\title{
Collective decision-making in living and artificial systems: editorial
}

\author{
Andreagiovanni Reina $^{1,2}$ (D) Eliseo Ferrante ${ }^{3,4} \cdot$ Gabriele Valentini $^{5}$
}

Received: 14 May 2021 / Accepted: 18 May 2021 / Published online: 3 June 2021

(c) The Author(s) 2021

Collective decision-making is a fundamental cognitive process required for group coordination. Typically, this process requires individuals in a group to either reach a consensus on one of several available options or to distribute their workforce over different tasks (Conradt and List 2009; Valentini et al. 2017). Similar collective decision-making processes can be found in a large number of systems, motivating a vast modeling effort across scientific disciplines (Bose et al. 2017). It can be observed across scales in a variety of animal groups, from unicellular organisms (Waters and Bassler 2005; Reid et al. 2016), to social insects (Seeley 2010; Camazine et al. 2003), fish schools (Lopez et al. 2012), and groups of mammals (Krause and Ruxton 2002; Strandburg-Peshkin et al. 2015). In the social sciences, scientific domains such as econophysics and sociophysics emerged to investigate collective decisions in humans, deepening our understanding of the dynamics of economies and social policies (Galam 2008; Molavi et al. 2018). Neuroscientists also look at brains as a collection of neurons that, through numerous interactions, lead to rational decisions (Bogacz et al. 2006; Usher and McClelland 2001; Bose et al. 2019). Studies of collective decision-making in nature inspired the engineering of decentralized cyber-physical systems such as robot swarms and wireless sensor networks with the potential to create new emerging and disruptive technologies (Valentini et al. 2017; Hamann 2018; Dorigo et al. 2021). Collective decision-making, ubiquitous across living and artificial collectives, can benefit from an interdisciplinary approach as apparently different systems may share similar mechanisms. With this special issue, we aim to push forward such an interdisciplinary approach by providing perspectives and insights from biology, information science, and engineering. The issue collects seven articles, out of 22 submissions received (acceptance rate $32 \%$ ), that have been accepted for publication after at least two rounds of reviews with comments by at least three referees.

Andreagiovanni Reina

andreagiovanni.reina@ulb.be

1 IRIDIA, Université Libre de Bruxelles, Brussels 1050, Belgium

2 Department of Computer Science, University of Sheffield, Sheffield S1 4DP, UK

3 Department of Computer Science, Vrije Universiteit Amsterdam, Amsterdam 1081HV, The Netherlands

4 Autonomous Robotics Research Centre, Technology Innovation Institute (ARRC-TII), Abu Dhabi, United Arab Emirates

5 School of Life Sciences, Arizona State University, Tempe, AZ 85287, USA 
So far, the design of algorithms for decentralized artificial systems has been largely inspired by studies of animal behavior in which a group of animals self-organize. Studies on the selection of nest sites (Seeley et al. 2012; Detrain and Deneubourg 2008) or foraging sites (Shaffer et al. 2013; Deneubourg and Goss 1989) as well as on how a common direction of motion is chosen (Couzin et al. 2005) led to the development of algorithms and models for logically analogous robotic systems for consensus agreement (Valentini 2017), allocation to resources (Talamali et al. 2020), and coordination of motion (Ferrante et al. 2012). In this context, a new frontier for artificial systems is to design algorithms capable to explicitly deal with spatial constraints (Dorigo et al. 2020). In "Nest choice in arboreal ants is an emergent consequence of network creation under spatial constraints," Joanna Chang, Scott Powell, Elva J. H. Robinson, and Matina C. Donaldson-Matasci provide the biological basis for new developments in this direction by investigating how spatially constrained pathways affect the selection of nest sites by turtle ants. These ants live in an arboreal environment and rely on available branches to form transport networks between multiple nests and foraging locations. The selection of proper nest sites requires therefore to account for the spatial constraints imposed by their environment. Chang and colleagues collected experimental evidence in the laboratory by varying the configuration of transport networks connecting different nest sites and subsequently developed an agent-based model to further explore the behavioral mechanisms underlying their observations. They found that simple random movements coupled with a constrained environment and a tendency of turtle ants to aggregate in higher density areas suffice to converge to a collective decision.

In "Enhanced or distorted wisdom of crowds? An agent-based model of opinion formation under social influence," Pavlin Mavrodiev and Frank Schweitzer warn us about possible side effects of social influence on the wisdom of crowds. Using an agent-based model and by varying the amount of information available to individual agents to make a decision, they show that social influence generally reduces the benefits of the wisdom of the crowd. The study of Mavrodiev and Schweitzer serves as an admonishment to engineers of artificial systems that new mechanisms capable to counterbalance the side effects of social influence are necessary.

The gap between studies of living and artificial collectives is typically bridged through the use of mathematical modeling. For example, irrespective of the nature of the collective system at hand, collective decision-making processes are generally based on a combination of positive and negative feedback loops. In "Analysis and control of agreement and disagreement opinion cascades," Alessio Franci, Anastasia Bizyaeva, Shinkyu Park, and Naomi E. Leonard analyze a generic model of collective decision-making based on excitatory and inhibitory feedback loops among opinions (Bizyaeva et al. 2020). Their mathematical model generalizes a large class of decentralized voting strategies studied in the literature and based on excitatory/inhibitory feedback loops (e.g., Gray et al. 2018; Altafini 2013; Seeley et al. 2012; Reina et al. 2017); it is therefore of interest both to researchers studying natural collectives and to those engineering artificial systems. The authors show that through certain simplifications and assumptions, their model can be mathematically analyzed via a small set of control parameters. By regulating the "attention" parameter, that indicates the tendency of an individual to align with or deviate from the preferences of its peers, a collective can converge to a consensus or split into sub-populations with polarized opinions. Through a combination of bifurcation and graph theory, Franci and colleagues also illustrate efficient methods to control the decision of a collective system composed of individuals that follow simple excitation and inhibition rules.

The mechanism through which individuals combine error-prone estimates of environmental features with social information received from their peers is key to make collective 
decisions. Simple reactive rules, such as excitation and inhibition, have the advantage of requiring limited cognitive abilities. The benefits of simple rules, however, are counterpoised by suboptimal collective performance. More advanced mechanisms, such as sophisticated fusion operators, have the potential to grant better collective performance at the cost of increased requirements at the individual level. In this special issue, two studies explore this research direction by designing robot swarms' behaviors based on fusion operators from epistemic logic in the context of the best-of- $n$ problem. In "Multi-featured collective perception with evidence theory: Tackling spatial correlations," Palina Bartashevich and Sanaz Mostaghim compare the performance of eight fusion operators from evidence theory (Dempster 1967; Shafer 1976) in the presence of highly variable noise. The authors consider several possible environments that differ in the type of noise experienced by individuals: from uniform noise throughout the entire environment, to highly heterogeneous levels of noise across different locations. Their results, obtained through agent-based simulations, show that swarms of limited size benefit the most by employing the fusion operator of "proportional conflict redistribution" (Smarandache and Dezert 2005). However, their results also evidence scalability limits of the tested fusion operators in the case of large swarms and for a large number of available options. A possible solution to scalability problems of fusion operations is proposed in "Negative updating applied to the best-of- $n$ problem with noisy qualities" by Chanelle Lee, Jonathan Lawry, and Alan F. T. Winfield. The authors show that probabilistic opinion pooling operators can be successfully applied in swarm robotics. They consider a site-selection problem and allow for robots in close proximity of a site to obtain a noisy estimate of the site quality. By means of real-robot experiments, they show that the negative update of evidence allows the swarm to correct individual errors and to efficiently select the best site among a large number of alternatives, even when the number of options is larger than the number of robots comprising the swarm.

Whereas most initial studies in swarm robotics focused on binary problems (Valentini et al. 2017), with only few examples considering a larger number of options (Reina et al. 2017; Lee et al. 2018; Talamali et al. 2019), we can appreciate in this special issue a shift in research focus as demonstrated by the studies of Franci et al., Lee et al., and Bartashevich and Mostaghim, discussed earlier. In "Collective preference learning in the bestof- $n$ problem: From best-of- $n$ to ranking $n$," Michael Crosscombe and Jonathan Lawry also pursue this research direction and propose the ranking of all available options as a viable and more efficient alternative to repeatedly solve a series of best-of- $n$ problems. By comparing the performance of a set of decentralized ranking algorithms over increasing levels of noise and numbers of options, they provide evidence of a trade-off between improved collective performance and lower computational costs at the level of the individuals.

In addition to reaching an agreement, the individuals of a group involved in a collective decision-making process must understand when the decision is made-a process often referred to as quorum sensing (Pratt et al. 2002; Waters and Bassler 2005). In "Quorum sensing without deliberation: Biological inspiration for externalizing computation to physical spaces in multi-robot systems"1, Theodore P. Pavlic, Jake Hanson, Gabriele Valentini, Sara I. Walker, and Stephen C. Pratt propose a quorum-sensing mechanism that does not require robots to accumulate information over time within their memory. Canonical implementations of quorum sensing are generally based on keeping track of encounters with

\footnotetext{
1 To guarantee a fair reviewing process, the paper by Pavlic et al. has been managed by the editor-in-chief of the Swarm Intelligence journal following the standard procedure for regular submissions because one of the authors of the paper is also a guest editor of this special issue.
} 
other swarm members with the aim to estimate an encounter rate and, on this basis, to decide when a quorum is met. Instead, Pavlic et al. propose a mechanism that does not require the circuitry necessary to implement a digital counter but externalizes such computation into the environment. Although the ability to count encounters can be taken as granted in most types of robots, this study contributes toward the challenge of designing collective decision-making in swarms of individuals with minimal computation abilities, such as, for example, future nano-robot swarms for medical applications (Yasa et al. 2020).

As demonstrated by the diversity of contributions in this special issue, collective decision-making is a research area whose success requires a highly interdisciplinary approach. By organizing this special issue, we aimed to foster such an interdisciplinary approach and to promote an exchange of consolidated concepts and new ideas across researchers from different scientific fields. Much research is still required to advance our understanding of the many ways a group of individuals can make collective decisions and we believe this issue can serve to motivate the scientific community in this direction.

Open Access This article is licensed under a Creative Commons Attribution 4.0 International License, which permits use, sharing, adaptation, distribution and reproduction in any medium or format, as long as you give appropriate credit to the original author(s) and the source, provide a link to the Creative Commons licence, and indicate if changes were made. The images or other third party material in this article are included in the article's Creative Commons licence, unless indicated otherwise in a credit line to the material. If material is not included in the article's Creative Commons licence and your intended use is not permitted by statutory regulation or exceeds the permitted use, you will need to obtain permission directly from the copyright holder. To view a copy of this licence, visit http://creativecommons.org/licenses/by/4.0/.

\section{References}

Altafini, C. (2013). Consensus problems on networks with antagonistic interactions. IEEE Transactions on Automatic Control, 58(4), 935-946. https://doi.org/10.1109/TAC.2012.2224251.

Bizyaeva, A., Franci, A., \& Leonard, N. E. (2020). A general model of opinion dynamics with tunable sensitivity. arXiv, math.OC(2009.04332).

Bogacz, R., Brown, E., Moehlis, J., Holmes, P., \& Cohen, J. D. (2006). The physics of optimal decision making: A formal analysis of models of performance in two-alternative forced-choice tasks. Psychological Review, 113(4), 700-765. https://doi.org/10.1037/0033-295X.113.4.700.

Bose, T., Reina, A., \& Marshall, J. A. R. (2017). Collective decision-making. Current Opinion in Behavioral Sciences, 6, 30-34. https://doi.org/10.1016/j.cobeha.2017.03.004.

Bose, T., Reina, A., \& Marshall, J. A. R. (2019). Inhibition and excitation shape activity selection: effect of oscillations in a decision-making circuit. Neural Computation, 31(5), 870-896. https://doi.org/10. 1162/neco_a_01185.

Camazine, S., Deneubourg, J.-L., Franks, N. R., Sneyd, J., Bonabeau, E., \& Theraulaz, G. (2003). Selforganization in biological systems. Princeton: Princeton University Press.

Conradt, L., \& List, C. (2009). Group decisions in humans and animals: A survey. Philosophical Transactions of the Royal Society B: Biological Sciences, 364(1518), 719-742. https://doi.org/10.1098/rstb. 2008.0276.

Couzin, I. D., Krause, J., Franks, N. R., \& Levin, S. A. (2005). Effective leadership and decision-making in animal groups on the move. Nature, 433(7025), 513-516. https://doi.org/10.1038/nature03236.

Dempster, A. P. (1967). Upper and lower probabilities induced by a multivalued mapping. The Annals of Mathematical Statistics, 38(2), 325-339. https://doi.org/10.1214/aoms/1177698950.

Deneubourg, J.-L., \& Goss, S. (1989). Collective patterns and decision-making. Ethology Ecology \& Evolution, $1,295-311$.

Detrain, C., \& Deneubourg, J.-L. (2008). Collective decision-making and foraging patterns in ants and honeybees. In S. Simpson (Ed.), Advances in insect physiology. Cambridge: Academic Press. 
Dorigo, M., Theraulaz, G., \& Trianni, V. (2020). Reflections on the future of swarm robotics. Science Robotics, 5(49), eabe4385. https://doi.org/10.1126/scirobotics.abe4385.

Dorigo, M., Theraulaz, G., \& Trianni, V. (2021). Swarm robotics: Past, present and future. Proceedings of the IEEE. (in press).

Ferrante, E., Turgut, A. E., Huepe, C., Stranieri, A., Pinciroli, C., \& Dorigo, M. (2012). Self-organized flocking with a mobile robot swarm: A novel motion control method. Adaptive Behavior, 20(6), 460477. https://doi.org/10.1177/1059712312462248.

Galam, S. (2008). Sociophysics: A review of Galam models. International Journal of Modern Physics C, 19(3), 409-440. https://doi.org/10.1142/S0129183108012297.

Gray, R., Franci, A., Srivastava, V., \& Leonard, N. E. (2018). Multiagent decision-making dynamics inspired by honeybees. IEEE Transactions on Control of Network Systems, 5(2), 793-806. https://doi. org/10.1109/TCNS.2018.2796301.

Hamann, H. (2018). Swarm robotics: A formal approach. Cham: Springer.

Krause, J., \& Ruxton, G. D. (2002). Living in groups. Oxford: Oxford University Press.

Lee, C., Lawry, J., \& Winfield, A. F. T. (2018). Negative updating combined with opinion pooling in the best-of-n problem in swarm robotics. In Swarm Intelligence: 11th International Conference, ANTS 2018, Rome, Italy, October 29-31, 2018, Proceedings, volume 11172, pages 97-108. Springer.

Lopez, U., Gautrais, J., Couzin, I. D., \& Theraulaz, G. (2012). From behavioural analyses to models of collective motion in fish schools. Interface Focus, 2(6), 693-707. https://doi.org/10.1098/rsfs.2012.0033.

Molavi, P., Tahbaz-Salehi, A., \& Jadbabaie, A. (2018). A theory of non-Bayesian social learning. Econometrica, 86(2), 445-490. https://doi.org/10.3982/ECTA14613.

Pratt, S. C., Mallon, E., Sumpter, D., \& Franks, N. R. (2002). Quorum sensing, recruitment, and collective decision-making during colony emigration by the ant Leptothorax albipennis. Behavioral Ecology and Sociobiology, 52(2), 117-127. https://doi.org/10.1007/s00265-002-0487-x.

Reid, C. R., MacDonald, H., Mann, R. P., Marshall, J. A. R., Latty, T., \& Garnier, S. (2016). Decision-making without a brain: How an amoeboid organism solves the two-armed bandit. Journal of The Royal Society Interface, 13(119), 20160030. https://doi.org/10.1098/rsif.2016.0030.

Reina, A., Marshall, J. A. R., Trianni, V., \& Bose, T. (2017). Model of the best-of- $N$ nest-site selection process in honeybees. Physical Review E, 95(5), 052411. https://doi.org/10.1103/PhysRevE.95.052411.

Seeley, T. D. (2010). Honeybee democracy. Princeton: Princeton University Press.

Seeley, T. D., Visscher, P. K., Schlegel, T., Hogan, P. M., Franks, N. R., \& Marshall, J. A. R. (2012). Stop signals provide cross inhibition in collective decision-making by honeybee swarms. Science, 335(6064), 108-111. https://doi.org/10.1126/science.1210361.

Shafer, G. (1976). A mathematical theory of evidence. Princeton: Princeton University Press.

Shaffer, Z., Sasaki, T., \& Pratt, S. C. (2013). Linear recruitment leads to allocation and flexibility in collective foraging by ants. Animal Behaviour, 86(5), 967-975. https://doi.org/10.1016/j.anbehav.2013.08. 014.

Smarandache, F.,\& Dezert, J. (2005). Information fusion based on new proportional conflict redistribution rules. In 2005 7th International Conference on Information Fusion, volume 2, page 8 pp. https://doi. org/10.1109/ICIF.2005.1591955.

Strandburg-Peshkin, A., Farine, D. R., Couzin, I. D., \& Crofoot, M. C. (2015). Shared decision-making drives collective movement in wild baboons. Science, 348(6241), 1358-1361. https://doi.org/10.1126/ science.aaa5099.

Talamali, M. S., Marshall, J. A. R., Bose, T.,\& Reina, A. (2019). Improving collective decision accuracy via time-varying cross-inhibition. In: Proceedings of the 2019 IEEE International Conference on Robotics and Automation (ICRA 2019), pages 9652-9659. IEEE. https://doi.org/10.1109/ICRA.2019.8794284.

Talamali, M. S., Bose, T., Haire, M., Xu, X., Marshall, J. A. R., \& Reina, A. (2020). Sophisticated collective foraging with minimalist agents: A swarm robotics test. Swarm Intelligence, 14(1), 25-56. https://doi. org/10.1007/s11721-019-00176-9.

Usher, M., \& McClelland, J. L. (2001). The time course of perceptual choice: The leaky, competing accumulator model. Psychological Review, 108(3), 550-592. https://doi.org/10.1037/0033-295X.108.3. 550 .

Valentini, G. (2017). Achieving consensus in robot swarms: Design and analysis of strategies for the bestof- $n$ problem. In Studies in computational intelligence (Vol. 706). Springer.

Valentini, G., Ferrante, E., \& Dorigo, M. (2017). The best-of-n problem in robot swarms: Formalization, state of the art, and novel perspectives. Frontiers in Robotics and AI. https://doi.org/10.3389/frobt. 2017.00009 .

Waters, C. M., \& Bassler, B. L. (2005). Quorum sensing: Communication in bacteria. Annual Review of Cell and Developmental Biology, 21(1), 319-346. https://doi.org/10.1146/annurev.cellbio.21.012704. 131001. 
Yasa, I. C., Ceylan, H., Bozuyuk, U., Wild, A.-M., \& Sitti, M. (2020). Elucidating the interaction dynamics between microswimmer body and immune system for medical microrobots. Science Robotics. https:// doi.org/10.1126/scirobotics.aaz3867.

Publisher's Note Springer Nature remains neutral with regard to jurisdictional claims in published maps and institutional affiliations. 